\title{
Concomitant herpetic keratitis and acute retinal necrosis: clinical features and outcomes
}

\author{
Wendy Ming $\mathbb{D}^{1} \cdot$ Nikhil Dewan $^{1,2} \cdot$ Sonia N. Yeung ${ }^{1} \cdot$ Alfonso lovieno $^{1}$
}

Received: 25 August 2019 / Revised: 12 February 2020 / Accepted: 13 February 2020 / Published online: 24 February 2020

(c) The Author(s), under exclusive licence to The Royal College of Ophthalmologists 2020

\begin{abstract}
Background/Objectives The aim of this study was to identify risk factors, prognosis, and management in patients with concomitant herpetic keratitis and acute retinal necrosis (ARN).

Subjects/Methods In this retrospective observational series, ARN patients in the Greater Vancouver area from 2004 to 2018 were identified through key words, billing codes, and dispensing data from medical records of affiliate researchers and the pharmacy at Vancouver General Hospital.

Results Twenty-eight patients were diagnosed with ARN during this period. Five of those were also diagnosed with herpetic keratitis. Thus, the incidence of combined keratitis and ARN was 18\% of patients with ARN. The two diseases occurred concomitantly in four out of five patients. Mean age at diagnosis was $55 \pm 17$ years. Of the five patients with both corneal and retinal involvement, three patients were immunocompromised and three had a history of herpetic diseases. Three developed disciform keratitis and two developed stromal immune keratitis. Clinical management and outcomes of these patients did not differ from patients with ARN alone, except for a lower rate of retinal detachment $(p=0.006)$ and a higher proportion with visual acuity $\geq 20 / 200(p=0.01)$.

Conclusions Keratitis may complicate the clinical picture of one out of five to six patients with ARN. Comprehensive ophthalmic examination, including careful anterior segment exams in patients with ARN and sequential dilated fundus exams in patients with herpetic keratitis, should always be conducted to identify cases of concomitant disease.
\end{abstract}

\section{Introduction}

Acute retinal necrosis (ARN) is thought to be due to reactivation of Herpesviridae, most frequently herpes simplex virus or herpes zoster virus (HSV/HZV), seated within the trigeminal ganglion. Based on two nationwide surveys of ophthalmologists in the United Kingdom (UK), the incidence of ARN is estimated at $0.5-0.63$ new cases per million population per year [1]. With the advent of polymerase chain reaction (PCR), anterior chamber or vitreous fluid is positive for $\mathrm{HSV} / \mathrm{HZV}$ in $79-100 \%$ of suspected ARN cases. Systemic antivirals, either oral or intravenous, remain first-line treatment. The addition of intravitreal foscarnet to systemic

Alfonso Iovieno

alfonsoiovieno@hotmail.com

1 Department of Ophthalmology, University of British Columbia, 2550 Willow Street, Vancouver, BC V5Z 3N9, Canada

2 Royal College of Surgeons in Ireland, Dublin, Ireland antivirals may have greater efficacy than systemic antivirals alone [2]. Adjunct therapies with unknown efficacy have also been tried, including topical steroids, systemic steroids, systemic antiplatelet agents, prophylactic barrier laser, and prophylactic vitrectomy [1,2]. Complications such as retinal detachment, cystoid macular oedema, and epiretinal membranes, along with surgeries for these complications, lead to further deterioration of vision [1].

Herpesviridae are also a common cause of keratitis. The estimated incidence of HSV keratitis ranges from 0.004 to $0.027 \%$, and the incidence of HZV keratitis from 0.013 to $0.053 \%[3,4]$. Corneal scarring affecting visual acuity may be treated with penetrating or deep anterior lamellar keratoplasty, although the incidence of keratitis recurrence in graft ranges from 6 to 47\% [5]. Long-term prophylaxis with oral antivirals such as acyclovir may reduce the rate of recurrence by up to $50 \%$ [6].

Although there have been case reports of herpetic keratitis associated with ARN, our study aimed to identify all cases of concomitant keratitis and ARN occurring in British Columbia over a 15-year timeframe and analysing their outcomes. 


\section{Materials and methods}

Our study conformed to the tenets of the Declaration of Helsinki and was approved by the University of British Columbia Clinical Research Ethics Board. The electronic medical record systems of affiliate investigators were searched for individuals with a clinical diagnosis of ARN from January 1, 2004 to July 13, 2018. The list of patients who were dispensed intravitreal foscarnet or ganciclovir over the same timeframe was also obtained from the pharmacy department at Vancouver General Hospital. Clinical charts of these patients were then reviewed to confirm the diagnoses. Demographics, corrected distance visual acuity (CDVA, Snellen), clinical features, treatment modalities, and complications were recorded for all patients.

Patients were divided into two groups for analysis: ARN alone or ARN with herpetic keratitis. Student's $t$ test and chi-squared test were used for comparisons, assuming approximately normal distribution, and similar variance.

\section{Results}

Twenty-eight patients with a clinical diagnosis of ARN in the last 15 years were included in the study. They ranged in age from 20.0 to 87.9 years old (mean $52.9 \pm 19.6$ years). Nineteen were male and nine were female. The incidence of ARN in our population was 0.45 per 1 million people per year. Of those with ARN, five patients were also diagnosed with herpetic keratitis. The incidence of both conditions was therefore 0.08 per 1 million people per year, or $18 \%$ of those diagnosed with ARN.

Characteristics of patients diagnosed with both keratitis and ARN are shown in Table 1. Of the five patients with keratitis and ARN, two (40\%) were male and three $(60 \%)$ were female. One patient developed bilateral ARN, but keratitis only in one eye (patient D). The rest developed unilateral disease, and a representative fundus photograph is shown in Fig. 1a (patient C). Three (60\%) were immunocompromised, two (40\%) were post-renal transplant, and one $(20 \%)$ had chronic kidney disease. Three of the five patients had previous herpetic diseases, including V1 zoster, disseminated zoster, and genital herpes. Four patients $(80 \%)$ were diagnosed concomitantly (i.e. within 1 week) with both herpetic keratitis and ARN. One patient $(20 \%)$ developed keratouveitis a year after the initial diagnosis of ARN. Three (60\%) patients had disciform keratitis (Fig. 1b), while the other two (40\%) developed stromal immune keratitis. In all cases, corneal findings resolved within 2 months. Aqueous samples of two patients $(40 \%)$ were PCR positive for $\mathrm{HZV}$, one (20\%) was negative, and two $(40 \%)$ had insufficient samples for analysis. All patients were treated with topical

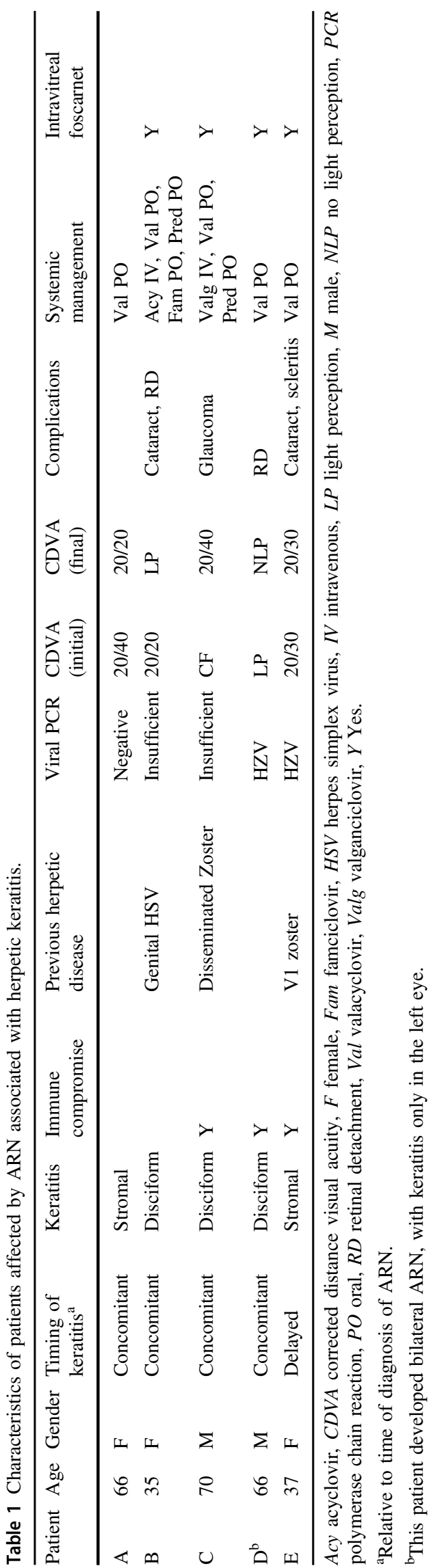


Fig. 1 Fundus and slit-lamp photographs of one patient with acute retinal necrosis and disciform keratitis. a Wideshowing vitritis with multiple foci of retinitis. b Slit-lamp photograph showing disciform keratitis with keratic precipitates, anterior chamber cells, and flare. field fundus photograph

Table 2 Comparison between herpetic keratitis and ARN vs. ARN alone.

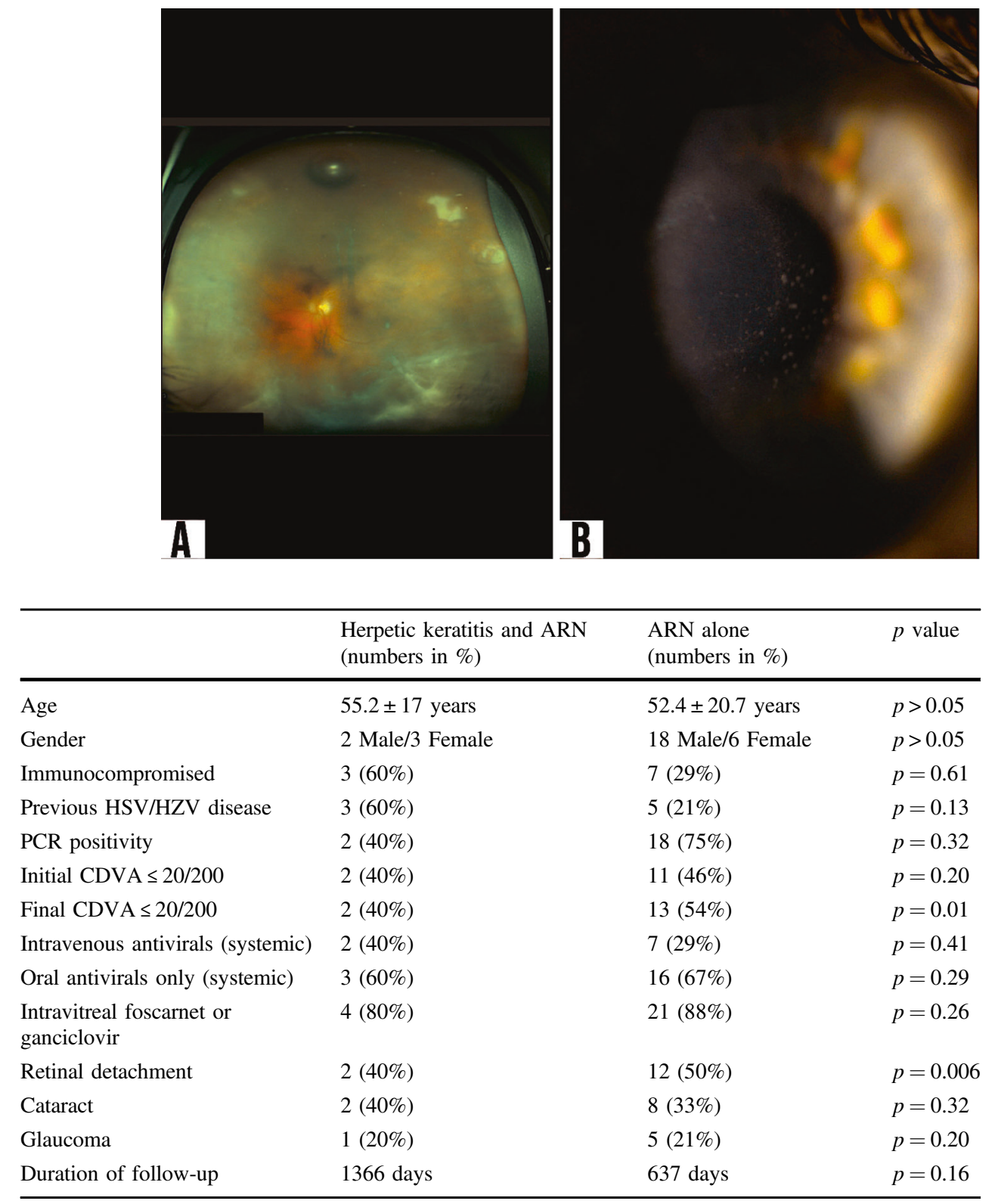

$A R N$ acute retinal necrosis, $C D V A$ corrected distance visual acuity, $H S V$ herpes simplex virus, $H Z V$ herpes zoster virus, $P C R$ polymerase chain reaction. steroids. All patients received systemic antiviral treatment for ARN. The most common treatment regimen was valacyclovir $500 \mathrm{mg}$ (renal compromise) to $2 \mathrm{~g}$ orally three times a day $(60 \%)$. Acute treatment duration ranged from 2 weeks to 6 months, with some patients continuing indefinitely at prophylactic doses. Two patients (40\%) also received intravenous acyclovir or valganciclovir for up to 7 days followed by oral antivirals. Four eyes $(80 \%)$ also received intravitreal foscarnet $2.4 \mathrm{mg} / 0.1 \mathrm{ml}$. One patient $(20 \%)$ was treated with systemic prednisone. Two patients (40\%) developed retinal detachments requiring laser or surgical management. Other complications included cataract (40\%), glaucoma (20\%), and scleritis (20\%).
Comparison between patients with keratitis and ARN vs. ARN only is shown in Table 2. There were no significant differences between age, gender, or immune status between those with herpetic keratitis and ARN vs. ARN alone. There were also no significant differences between the proportion of individuals with previous herpetic diseases, PCR positivity in aqueous or vitreous, initial or final CDVA $\geq 20 / 200$, or antiviral treatment modality. The most common treatment regimen in ARN alone was valacyclovir 1-2 mg orally TID for several months, with gradual tapering off the medication over up to a year $(52 \%)$. Three patients $(13 \%)$ planned to continue prophylactic doses of valacyclovir indefinitely. Seven patients $(30 \%)$ received intravenous 
antivirals for up to 10 days, six (26\%) with acyclovir, and one $(4 \%)$ with ganciclovir. Sixteen $(67 \%)$ and five eyes $(21 \%)$ received at least one dose of foscarnet $2.4 \mathrm{mg} / 0.1 \mathrm{ml}$ or ganciclovir $2 \mathrm{mg} / 0.1 \mathrm{ml}$ respectively. In addition, there was no difference between the rates of cataracts and glaucoma between the two groups. However, the rate of retinal detachment was significantly higher in those with ARN alone compared with those with both diseases.

\section{Discussion}

This study is the first to look at the incidence, clinical features, and outcomes of herpetic keratitis in association with ARN. The incidence of ARN in our study population was 0.45 per one million people per year. The incidence of ARN has previously been estimated to be between 0.5 and 0.63 cases per year in two large-scale UK studies, which is similar to our study $[1,7]$. Of the patients with ARN, 18\% (or one in five to six) were also found to have keratitis, and this is the first documented estimate of the incidence of both conditions.

The precise mechanism underlying the development of both conditions is unknown. The development of ARN after keratitis has been hypothesized to occur due to viral spread from the cornea to the ciliary ganglion through the Edinger-Westphal nucleus to the suprachiasmatic nucleus, and then through the optic nerve to the retina. It has been proposed that the reverse also might occur, causing keratitis in those with ARN. Direct invasion of the virus through the cornea, however, is thought to be unlikely due to Descemet's membrane, which is difficult for the virus to penetrate [8].

In our study, four of the five patients were diagnosed with keratitis and ARN within the span of a few days. This is consistent with previous case reports, where ARN and keratitis were diagnosed within a few weeks of onset of either disease [8,9]. One subject $(20 \%)$ in our study was diagnosed with keratitis much later than she was diagnosed with ARN, separated by a span of 11 months (patient E). Her keratitis presentation consisted of stromal haze consistent with stromal immune keratitis and minimal nongranulomatous anterior chamber reaction. Her ARN was stable without active posterior segment inflammation at the time of her keratitis diagnosis. Of the five patients diagnosed with both diseases, two had stromal immune keratitis. This has not been previously described to be associated with ARN. The other three patients $(60 \%)$ developed disciform keratitis, which has been documented to occur with ARN [10]. One patient $(20 \%)$ developed keratitis of one eye followed by ARN in both eyes. No patient developed epithelial keratitis in our study, although this has been observed in other case reports [8,9]. In all cases in our study, keratitis resolved without significant sequalae or scarring within 2 months [8-10].

In this study, two of the five patients with concomitant disease were PCR positive for HZV DNA in the aqueous humour (patients D and E, 40\%). Although HZV has been documented in previous case reports to cause concomitant disease [8], HSV-1 and HSV-2 have also been implicated [9, 11]. In our series, HZV was also the most common causative virus of ARN (59\%), followed by HSV-2 (23\%), and then by HSV-1 (9\%). The percentage of HZV in ARN in our study is slightly higher than in previous studies, which shows $\sim 50 \%$ positivity for $\mathrm{HZV}$ $[1,7,12]$. This may be due to a slightly higher proportion of patients with previous or concomitant zoster-related illnesses.

Three of five $(60 \%)$ patients with keratitis and ARN were immunocompromised, whereas only seven out of 24 (29\%) patients with ARN alone were immunocompromised. This difference was not statistically significant, likely due to the small number of patients with concomitant disease. Systemic conditions classified as causing an immunocompromised state included renal transplant (14\%), diabetes (7\%), malignancy $(7 \%)$, chronic kidney disease $(4 \%)$, bullous pemphigoid (4\%), and multiple sclerosis (4\%). ARN is well documented in immunocompetent and immunocompromised individuals, including those with renal transplant [13], multiple sclerosis [14], and human immunodeficiency virus [15]. Previous case reports for patients with both diseases also included immunocompetent patients [10, 11]. However, several of these reports do not mention the patients' immune status or past medical history [8-10].

Of the five patients with keratitis and ARN, three (60\%) had either HSV or HZV-related illnesses preceding the development of ARN. In the ARN alone group, only five out of 24 (22\%) patients had documented history of previous clinical herpetic infections. This is concordant with several case reports in the literature, noting a strong correlation between herpetic illnesses, such as V1 zoster dermatitis, herpetic meningitis, and herpetic encephalitis, and the development of ARN [16-18].

Our findings may therefore suggest that preceding herpetic infections and immunosuppression are risk factors for the development of combined herpetic keratitis and ARN, although there is insufficient statistical power to establish such correlation. We also describe an association between stromal immune keratitis and ARN, which has not previously been documented in literature.

Between patients with keratitis and ARN versus those with ARN alone, no significant differences were noted in demographics, clinical features, or management. However, there was a significant difference in the proportion of individuals with final CDVA of 20/200 or worse and retinal 
detachments between the two groups. Indeed, the final visual acuity of patients showed a bimodal distribution related to the final retinal status. Of the five patients with both conditions, two (40\%) experienced retinal detachments while three $(60 \%)$ did not. Those with retinal detachments did poorly with CDVA of light perception or worse, while those without attained CDVA of 20/40 or better. In the ARN alone group, 12 of 24 eyes $(50 \%)$ developed retinal detachments. Of these, four eyes achieved CDVA of 20/70 or better $(33 \%)$, while the rest $(67 \%)$ did poorly with CDVA of 20/200 or worse. This is consistent with research conducted by Butler et al., which demonstrated similar rates of retinal detachment in ARN, and identified retinal detachment as being the most important determinant of final visual acuity [18]. With regards to the significantly higher rate of retinal detachment in those with ARN alone versus ARN with keratitis, we can only hypothesize that those with both conditions developed less severe retinitis. However, this difference in the rate of retinal detachment may simply be a consequence of the low number of patients with both conditions.

There are several limitations to this retrospective study. Some cases of ARN might have been missed over such a long time period. However, virtually all cases of ARN are referred to tertiary care centres in the Greater Vancouver Area in British Columbia due to difficulty with access to compounding facilities for intrvitreal antivirals outside of these centres. In addition, subjects with keratitis and ARN were followed for almost twice as long compared with those with ARN alone. This difference was not statistically significant; however, longer surveillance of the ARN alone group may result in an increase in the number of keratitis diagnoses. Furthermore, it is difficult to meaningfully generate and interpret statistics due to the rarity of patients with both diseases. Nonetheless, this study provides the first series of combined ARN and herpetic keratitis identified in a large data set over a long period of time. The two diseases seemed to occur more commonly in immunocompromised individuals or immediately following herpesrelated diseases. Further studies are needed to shed some light on the potential mechanism for the development of both diseases. This study highlights the need for careful, serial, examinations of the anterior and posterior segment. Finally, clinicians should have a heightened awareness for the possibility of patients developing both conditions, particularly the risk of subtle keratitis in patients with ARN.

\section{Summary}

\section{What was known before}

- ARN is a relatively rare condition (less than 0.63 person per million) and is associated with poor clinical outcome.

\section{What this study adds}

- Analysis of 15 years of data demonstrated that $18 \%$ of patients with ARN also develop herpetic keratitis.

- Herpetic keratitis may be diagnosed before, at the same time, or after the diagnosis of ARN.

- Clinical outcomes of patients with keratitis and ARN do not differ from those with retinal necrosis alone.

Acknowledgements We would like to thank Dr D. Albiani, Dr D. Dhanda, Dr F. Forooghian, Dr A. Joe, Dr A. Kirker, Dr S. Lam, Dr L. Levasseur, Dr P. Ma, Dr D. Maberley, Dr A. Merkur, Dr E. Navajas, Dr H. Parsons, and Dr L. Wittenberg for their support.

\section{Compliance with ethical standards}

Conflict of interest The authors declare that they have no conflict of interest.

Publisher's note Springer Nature remains neutral with regard to jurisdictional claims in published maps and institutional affiliations.

\section{References}

1. Muthiah MN, Michaelides M, Child CS, Mitchell SM. Acute retinal necrosis: a national population-based study to assess the incidence, methods of diagnosis, treatment strategies and outcomes in the UK. Br J Ophthalmol. 2007;91:1452-5.

2. Schoenberger SD, Kim SJ, Thorne JE, Mruthyunjaya P, Yeh S, Bakri SJ, et al. Diagnosis and treatment of acute retinal necrosis. Ophthalmology. 2017;124:382-92.

3. Young RC, Hodge DO, Liesegang TJ, Baratz KH. Incidence, Recurrence, and outcomes of herpes simplex virus eye disease in Olmsted County, Minnesota, 1976-2007. Arch Ophthalmol. 2010;128:1178-83.

4. Borkar DS, Tham VM, Esterberg E, Ray KJ, Vinoya AC, Parker JV, et al. Incidence of herpes zoster ophthalmicus: results from the Pacific ocular inflammation study. Ophthalmology. 2013;120:451-6.

5. Suresh PS, Tulo AB. Herpes simplex keratitis. Indian J Ophthalmol. 1999;47:155-65.

6. Herpetic Eye Disease Study Group. Acyclovir for the prevention of recurrent herpes simplex virus eye disease. N Engl J Med. 1998;339:300-6.

7. Cochrane TF, Silvestri G, Mcdowell C, Foot B, McAvoy CE. Acute retinal necrosis in the United Kingdom: results of a prospective surveillance study. Eye. 2012;26:370-7.

8. Ansari WH, Pichi F, Pecen PE, Lowder CY, Srivistava SK. Herpes zoster keratitis development after acute retinal necrosis. Int Ophthalmol. 2018;38:829-32.

9. Sado K, Kimura T, Hotta Y, Sakuma H, Hayakawa M, Kato K, et al. Acute retinal necrosis syndrome associated with herpes simplex keratitis. Retina. 1994;14:260-3.

10. Attia S, Khochtali S, Mbarek S, Amor SB, Zaouali S, Messaoud $\mathrm{R}$, et al. Acute retinal necrosis associated with central retinal artery occlusion followed after its resolution by keratouveitis. Ocul Immunol Inflamm. 2013;21:405-7.

11. Smith LK, Kurz PA, Wilson DJ, Flaxel CJ, Rosenbaum JT. Two patients with the von szily reaction: herpetic keratitis and contralateral retinal necrosis. Am J Ophthalmol. 2007;143:536-8. 
12. Butler NJ, Moradi A, Salek SS, Burkholder BM, Leung TG, Dunn JP, et al. Acute retinal necrosis: presenting characteristics and clinical outcomes in a cohort of polymerase chain reaction-positive patients. Am J Ophthalmol. 2017;179:179-89.

13. Chiang E, Pyatetsky D. Acute retinal necrosis secondary to varicella zoster virus in an immunosuppressed post-kidney transplant patient. Clin Med Res. 2012;10:240-1.

14. Sheikh Z, Jain S, Hillen M. Acute retinal necrosis in multiple sclerosis: a neuroimmunologic challenge! Neurology. 2016;86:972-3.

15. Ning BK, Kelly SP, Chu C, Morgan E. Sequential retinal necrosis secondary to varicella zoster in unrecognised long-standing HIV infection: patient safety report. BMJ Case Rep. 2018;2018:pii: bcr-2017-222237.

16. Yeo JH, Pepose JS, Stewart JA, Sternberg P Jr, Liss RA. Acute retinal necrosis syndrome following herpes zoster dermatitis. Ophthalmology. 1986;93:1418-22.

17. Vandercam T, Hintzen RQ, Boer JHD, Van der Lelij A. Herpetic encephalitis is a risk factor for acute retinal necrosis. Neurology. 2008;71:1268-74.

18. Hirota $\mathrm{K}$, Akimoto $\mathrm{M}$, Katsura T. Bilateral acute retinal necrosis after herpetic meningitis. Clin Ophthalmol. 2012;6: $551-3$. 